\title{
Efeito da bandagem elástica na ativação muscular e torque isométrico dos extensores do joelho em indivíduos com osteoartrite
}

\author{
Bruna Carvalho CARDOSO* \\ Nathália Lobato PIMENTEL** \\ Natane Ceccatto BELLEI** \\ Danielle Miyuki NISHIMOTO** \\ Marcelo Tavella NAVEGA*** \\ Deborah Hebling SPINOSO****
}

*Faculdade de

Medicina de Marilia,

Marilia, SP, Brasil.

${ }^{* *}$ Curso de Fisioterapia

da Universidade

Estadual Paulista "Júlio

de Mesquita Filho",

Campus de Marilia,

Marília, Brasil.

${ }^{* *}$ Departamento

de Fisioterapia e

Terapia Ocupacional,

Universidade Estadual

Paulista "Júlio de

Mesquita Filho",

Campus Marilia,

Marilia, SP, Brasil.

****Universidade

Estadual Paulista "Júlio de Mesquita Filho",

Campus Rio Claro, Rio

Claro, SP, Brasil.

\section{Resumo}

A osteoartrite é uma doença articular crônica, caracterizada por uma degeneração progressiva da cartilagem articular, que resulta em incapacidade funcional. Entre as articulações acometidas, o joelho é a mais afetada devido à sobrecarga mecânica imposta a essa articulação nas atividades diárias. A osteoartrite de joelho é considerada uma das formas clínicas mais incapacitantes da doença. 0 objetivo deste estudo foi verificar 0 efeito da bandagem elástica (BE) na ativação muscular do quadríceps e no torque isométrico dos extensores de joelho em individuos com Osteoartrite de Joelho. Participaram do estudo 13 voluntárias $(64,5 \pm 7,2$ anos) do sexo feminino, com diagnóstico radiológico de osteoartrite de joelho. Os procedimentos de coleta de dados foram realizados em três dias, com intervalo de sete dias entre eles. As voluntárias foram avaliadas em três diferentes condições: sem BE, BE placebo e BE ativação. Foram realizadas as avaliações do torque isométrico extensor do joelho e ativação eletromiográfica dos músculos reto femoral, vasto medial e vasto lateral por meio da eletromiografia de superfície. Para análise estatística foi adotado o teste ANOVA Medidas Repetidas, considerando nivel de significância de $p<0,05$. Os resultados mostraram que não houve diferença significativa para comparação da ativação muscular e torque entre as condições analisadas $(p=0,556)$. Conclui-se que a BE não foi capaz de promover uma alteração no recrutamento muscular e torque isométrico de quadriceps em indivíduos com osteoartrite de joelho.

PalaVRas-Chave: Eletromiografia; Fisioterapia; Força muscular; Articulações.

\section{Introduçãóa,b}

A osteoartrite é uma doença articular crônica, caracterizada por uma degeneração progressiva da cartilagem articular, que resulta em incapacidade funcional ${ }^{1}$. A apresentação clínica inclui dor no joelho, crepitação com o movimento articular e inflamação localizada, bem como distúrbios funcionais, tais como fraqueza muscular do quadríceps femoral ${ }^{1}$. Essa doença pode afetar 
praticamente $100 \%$ da população com mais de 60 anos de idade, apresentando importância significativa dentro dos serviços de saúde pública por ser uma das causas mais frequentes de dor no sistema musculoesquelético, aposentadoria precoce, além de causar incapacidade e afastamento do trabalho ${ }^{2,3}$.

Entre as articulaçóes acometidas, o joelho é a mais afetada devido à sobrecarga mecânica imposta a essa articulação nas atividades diárias ${ }^{2,3}$. A osteoartrite de joelho (OAJ) é considerada uma das formas clínicas mais incapacitantes da doença e $25 \%$ dos seus portadores não podem executar as principais tarefas habituais ${ }^{1,3}$.

Um aspecto comum entre indivíduos com OAJ é a fraqueza do músculo quadríceps ${ }^{1,4}$. Esse músculo apresenta um importante papel na manutenção da mobilidade e reflete a capacidade do indivíduo realizar tarefas como: andar, levantar e sentar da cadeira e subir e descer escadas ${ }^{5}$. Além disso, esses indivíduos apresentam redução de cerca de $60 \%$ do torque máximo dos extensores do joelho, possivelmente resultado da atrofia por desuso, secundária a dor e inibição artrogênica, que é definida como uma inabilidade do sistema central de ativar completamente todas as unidades motoras de um músculo durante o esforço voluntário ${ }^{6}$.

Neste caso, a instabilidade articular, a dor e o fluido intra-articular excessivo sensibilizam os mecanorreceptores capsulares que emitem sinais para interneurônios inibitórios medulares que vão inibir os motoneurônios alfa e, consequentemente, os sinais que seriam transmitidos ao quadríceps ${ }^{4}$. Além disso, as mudanças bioquímicas e metabólicas na síntese e degradação do colágeno do joelho osteoartrítico contribuem também para a alteração do controle motor ${ }^{4}$. Assim, indivíduos com OAJ teriam menor capacidade de ativação e geração de torque dos extensores de joelho do que indivíduos sadios ${ }^{4}$.

\section{Método}

\section{Sujeitos}

Os critérios de elegibilidade desse estudo foram: ser do gênero feminino; ter idade entre 50-75 anos; capazes de deambular sem auxílio de dispositivos
Apesar de ser uma doença crônica que não tem cura, sabe-se que hoje é possível modificar seu curso evolutivo, tanto em relação ao tratamento sintomático quanto ao seu prognóstico ${ }^{7}$. Entre os recursos terapêuticos utilizados para tratamento dessa patologia, a bandagem elástica $(\mathrm{BE})$ pode ser uma estratégia de intervenção que proporcione resultados imediatos.

De acordo com KaSE et al. (1996, 2003), a Kinesio Taping é uma bandagem elástica com massa adesiva $100 \%$ acrílico hipoalérgico, sensível ao calor e não contém qualquer substância química ou medicinal impregnada, além disso sua espessura e peso são similares ao da pele, contendo marcas na sua face adesiva que simulam os veios existentes na pele humana ${ }^{8,9}$. A bandagem pode ser aplicada com objetivo de diminuir a dor e edema, aumentar a estabilidade da articulação e melhorar o desempenho muscular ${ }^{8}$.

Os mecanismos de ação da $\mathrm{BE}$ ainda não foram completamente elucidados ${ }^{10}$. Alguns pesquisadores afirmam que as suas características adesivas e elásticas poderiam aumentar o espaço intersticial, favorecendo o fluxo sanguíneo e linfático além de ocasionar um estímulo excitatório na musculatura envolvida, facilitando o recrutamento muscular e consequentemente a capacidade de gerar força ${ }^{8}$.

Diante disso, a realização de uma avaliação eletromiográfica, que permite analisar o comportamento neuromuscular, em pacientes com OAJ durante a utilização da BE pode oferecer subsídios para compreensão dos efeitos terapêuticos proporcionados pelo uso dessa na musculatura extensora de joelho. Nessa perspectiva, o objetivo do presente estudo foi verificar a eficácia da $\mathrm{BE}$ na ativação dos músculos do quadríceps e torque isométrico dos extensores de joelho. Hipotetizamos que o uso da BE excitatória proporcione aumento do recrutamento muscular e torque em indivíduos com OAJ.

de marcha; não apresentarem outras patologias que levem a um comprometimento motor, artroplastia total ou parcial de joelho, lesôes nos membros inferiores nos últimos seis meses pregressos ao estudo ou outras doenças que impossibilitem a 
execução dos testes; não terem feito aplicação intraarticular de corticoides nos três meses prévios ao estudo. Além disso, as voluntárias não poderiam apresentar, no dia da avaliação, queixa de dor superior a $3 \mathrm{~cm}$ de acordo com a Escala Visual Analógica (EVA).

Participaram do estudo 13 voluntárias $(64,5 \pm 7,2$ anos) recrutadas na comunidade, com diagnóstico radiológico de classificação II e III, com base na escala de graduação radiológica em Osteoartrose de Kelgren-Lawrence ${ }^{12}$ (4 grau II e 9 grau III) e tempo médio de diagnóstico da doença de 4 anos.

Quanto ao uso de medicamentos, 5 voluntárias usavam medicamento com princípio ativo de glicosamina e nenhuma fazia uso de antiinflamatório no período da avaliação.

O tamanho amostral foi determinado baseado em dados de estudo piloto e foi utilizado o programa $\mathrm{G}^{*}$ Power, considerando como variável de desfecho a eletromiografia (EMG) do vasto lateral durante o teste de força dos extensores de joelho (poder $=0,80$, tamanho do efeito $=0,85$, erro $\alpha=$ $0,05)^{13}$. A TABELA 1 apresenta as características dos sujeitos.

Todas as participantes assinaram o termo de consentimento livre e esclarecido e o projeto foi aprovado pelo Comitê de Ética em Pesquisa local (parecer número: 1.299.741/2015).

TABELA 1 - Características dos sujeitos

\begin{tabular}{lc}
\hline Variável & $\begin{array}{c}\text { Média } \pm \text { DP } \\
(\boldsymbol{n}=\mathbf{1 3})\end{array}$ \\
\hline Idade (anos) & $64,50 \pm 7,25$ \\
\hline Massa $(\mathbf{K g})$ & $79,24 \pm 17,05$ \\
\hline Altura (m) & $1,59 \pm 0,09$ \\
\hline IMC $\left(\mathbf{K g m}^{-2}\right)$ & $31,58 \pm 4,99$ \\
\hline
\end{tabular}

\section{Procedimentos}

Os procedimentos de coleta de dados foram realizados em três dias, com intervalo de 7 dias entre eles ${ }^{14}$. No primeiro dia foi realizada anamnese e registrados os dados antropométricos para caracterização da amostra e avaliação do nível de dor por meio da Escala Visual Analógica (EVA), que consiste em uma linha de $10 \mathrm{~cm}$, não numerada, onde em uma extremidade é indicada a demarcação "sem dor", e na outra, "pior dor possível". Para quantificar a dor, as voluntárias foram orientadas a marcar na escala o quanto de dor elas frequentemente sentem durante as atividades de vida diária e a dor sentida no momento da avaliação. Além disso as voluntárias foram avaliadas em relação à força muscular dos extensores do joelho e a ativação eletromiográfica do músculo quadríceps. Anteriormente a realização desses testes, os indivíduos foram familiarizados com a avaliação do torque isométrico de joelho (item 2.2.1).
As voluntárias foram avaliadas nos testes acima citados em três diferentes condições: sem $\mathrm{BE}, \mathrm{BE}$ placebo e $\mathrm{BE}$ ativação. A sequência da avaliação foi definida por meio de aleatorizaçáo dirigida.

\section{Avaliação da força muscular dos extensores de joelho}

A avaliação da força muscular dos extensores de joelho foi realizada no membro inferior acometido. Inicialmente, as voluntárias realizaram aquecimento em uma bicicleta ergométrica horizontal (BIOTEC $2100^{\circledR}$ ) durante cinco minutos ${ }^{15}$. Antes do início do protocolo de avaliação foi feita uma familiarização com o equipamento composta por duas contraçôes submáximas do grupo muscular a ser avaliado ${ }^{16}$. Entre a familiarização e o início dos procedimentos de coleta de dados houve um intervalo de 5 minutos afim de evitar a fadiga do grupo muscular avaliado. 
O protocolo de avaliação consistiu de três contraçôes isométricas voluntárias máximas para o movimento de extensão da articulação do joelho, por um período de cinco segundos, com intervalo de trinta segundo entre cada contraçáa ${ }^{17}$.

Para avaliação da articulaçáo do joelho, a voluntária foi posicionada sentada na cadeira extensora com flexão de quadril e joelho a $90^{\circ}$
( $0^{\circ}$ extensão completa) e o eixo articular da cadeira alinhado com eixo articular do joelho. A alavanca foi posicionada acima dos maléolos lateral/medial do membro a ser avaliado e fixada no angulo de $90^{\circ}$ durante toda execução do teste. O tronco e o membro inferior contralateral foram estabilizados por cintos. A FIGURA 1 ilustra o posicionamento da voluntária.

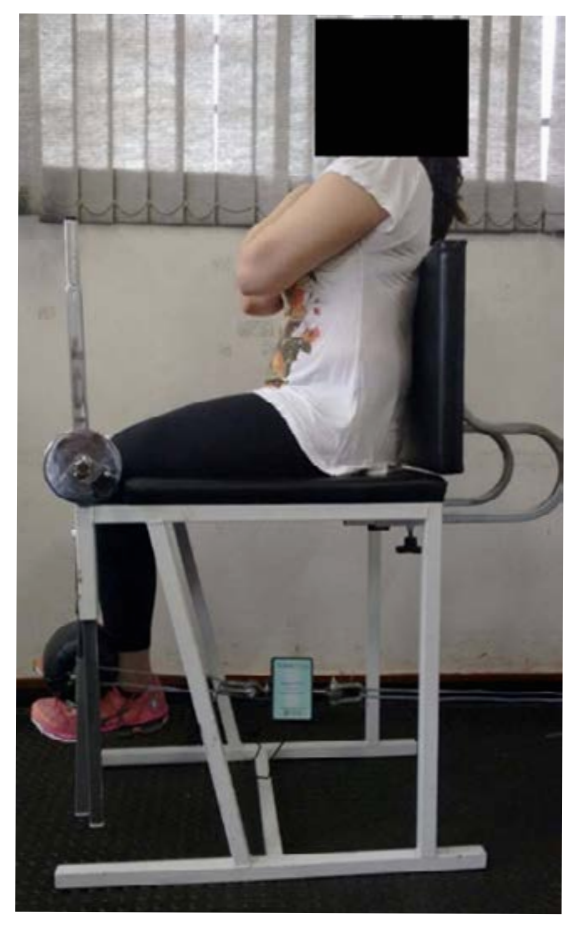

FIGURA 1 - Posicionamento da voluntária para avaliação da força muscular dos extensores de joelho

A célula de carga foi acoplada a alavanca da cadeira extensora para aquisição dos valores de torque muscular, utilizando um canal do equipamento EMG System, com saída entre 0 a $20 \mathrm{mV}$ e alcance até $1 \mathrm{kN}$ (EMG System do Brasil Ltda ${ }^{\circ}$ ).

O cálculo do torque extensor do joelho foi calculado a partir da seguinte equação:

Torque extensor de joelho = Força $(\mathrm{N})$ x Distância $x$ $\operatorname{sen} 90^{\circ} 18$

Onde, Distância $=1$ metro, $\operatorname{sen} 90^{\circ}=1$.

\section{Eletromiografia}

Para avaliação da ativação eletromiográfica dos músculos reto femoral (RF), vasto medial (VM) e vasto lateral (VL), foi realizado seu registro durante o teste de força muscular máxima. Para a captação do sinal eletromiográfico foi utilizado o sistema de aquisição com 8 canais (EMG System do Brasil Ltda ${ }^{\circledR}$ ), filtro analógico passa banda de 20 a $500 \mathrm{~Hz}$ e modo comum de rejeição $>100 \mathrm{~dB}$. Os sinais eletromiográficos amostrados com frequência de $2000 \mathrm{~Hz}$, digitalizados por placa de conversão A/D (analógico-digital) com 16 bits de resolução, e armazenados em disco para posterior análise. $\mathrm{O}$ software $\mathrm{EMGLab}^{\oplus}$ foi utilizado para análise do sinal.

Foram utilizados eletrodos de superfície de $\mathrm{Ag} / \mathrm{AgCl}$, em configuração bipolar, com área de captação de $1 \mathrm{~cm}$ de diâmetro e distância intereletrodos de $2 \mathrm{~cm}$. Previamente a colocaçáo dos eletrodos, foi realizada a tricotomia e limpeza da pele com álcool, como forma de evitar possíveis interferências no sinal eletromiográfico ${ }^{19}$.

Os eletrodos foram fixados no membro inferior acometido sobre os músculos: RF, VM e VL segundo as normas SENIAM ${ }^{20}$, como pode ser observado na FIGURA 2. 


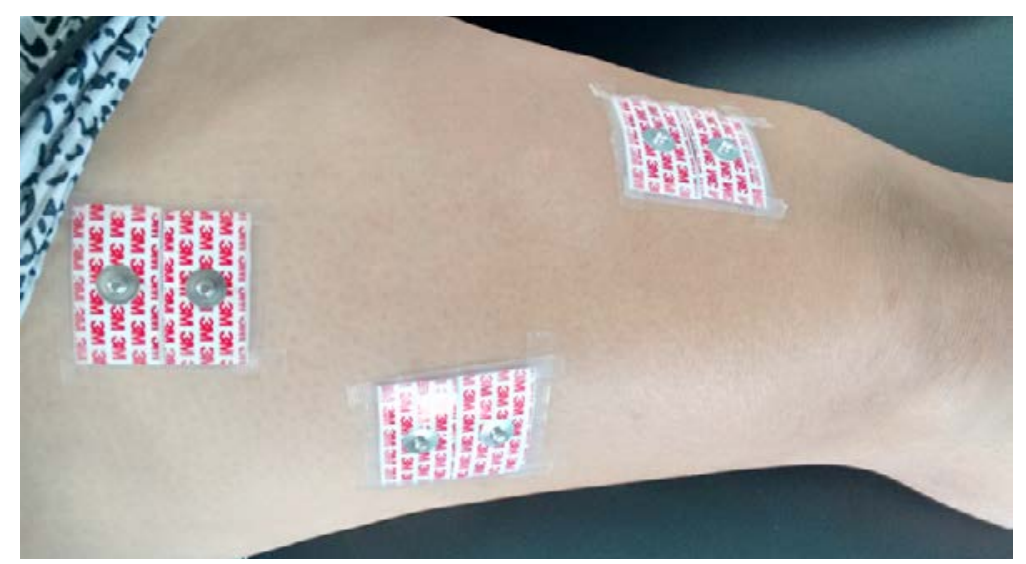

FIGURA 2 - Posicionamento dos eletrodos sobre os músculos reto femoral (RF), vasto medial (VM) e vasto lateral (VL)

\section{Aplicaçáo da bandagem elástica}

Foi utilizada a bandagem da marca KinesioSport elastic tape, a qual foi aplicada no músculo quadríceps conforme sugerido no estudo de Anandkumar et al. (2014), utilizando as técnicas propostas por KASE, et al. $(2003)^{8,11}$.

Para a aplicação terapêutica da BE, três tiras em "I " foram retiradas e aplicadas como se mostra na FIGURA 3. A base da primeira tira foi aplicada $10 \mathrm{~cm}$ abaixo da espinha ilíaca ântero-superior. Em seguida, a bandagem foi aplicada com tensão submáxima ao longo do curso do RF até a borda superior da patela. O joelho foi então flexionado a $45^{\circ} \mathrm{com}$ a bandagem restante aplicada sem tensão, se estendendo ao longo da borda superior da patela.A base da tira do segundo "I " foi aplicada abaixo do trocânter maior e a bandagem foi aplicada com tensão submáxima ao longo do curso do VL até a borda lateral da patela. Em seguida, o joelho foi novamente flexionado e a bandagem aplicada sem tensão em direção à tuberosidade anterior da tíbia.
A base da terceira tira foi aplicada a partir do meio $1 / 3$ da parte medial da coxa. A bandagem foi aplicada com uma tensão submáxima ao longo do curso do VM em direçáo à borda medial da patela. $\mathrm{O}$ joelho foi flexionado e a bandagem restante foi aplicada sem tensão em torno da borda medial da patela. Após a aplicação, o papel foi friccionado para ativar a cola ${ }^{8}$. A tensão na bandagem foi gerada no sentido da origem para a inserção muscular para faciliar o recrutamento muscular, conforme recomendaçóes de KASE et al. (2003) ${ }^{8}$.

Para a condição placebo, a aplicação da bandagem foi feita com as três tiras em "I " sem tensão, aplicadas nas mesmas posições anteriormente relatadas, mas sem respeitar a distribuição anatômica correta, sendo aplicada do centro para as extremidades.

Para aplicação simultânea dos eletrodos e captação do sinal eletromiográfico, foram realizadas fendas na bandagem ${ }^{8,9}$. Após a aplicação, as voluntárias aguardaram um período de 30 $\min ^{11}$. Feito isso, os testes foram realizados. A FIGURA 4 mostra como foi o fluxo dos participantes durante o estudo.

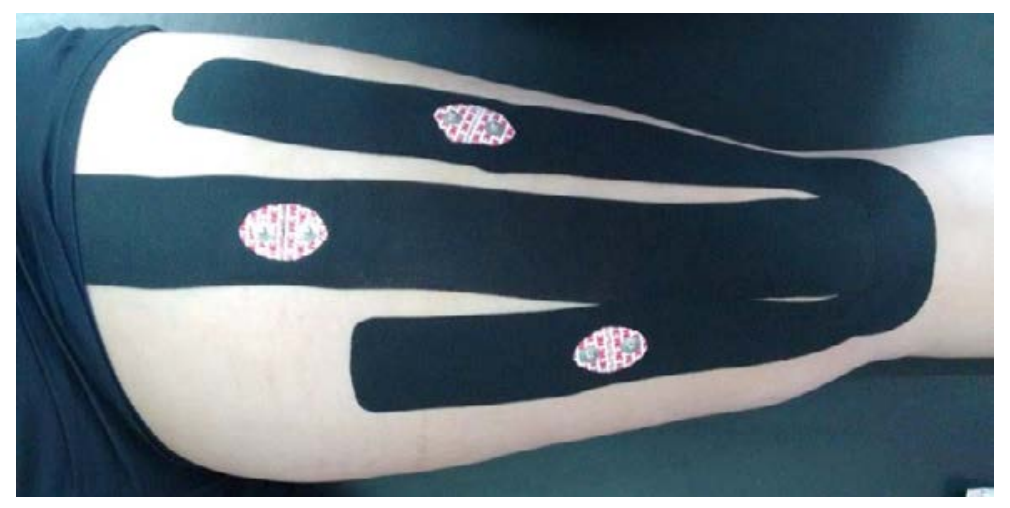

FIGURA 3 - Aplicação da bandagem elástica 


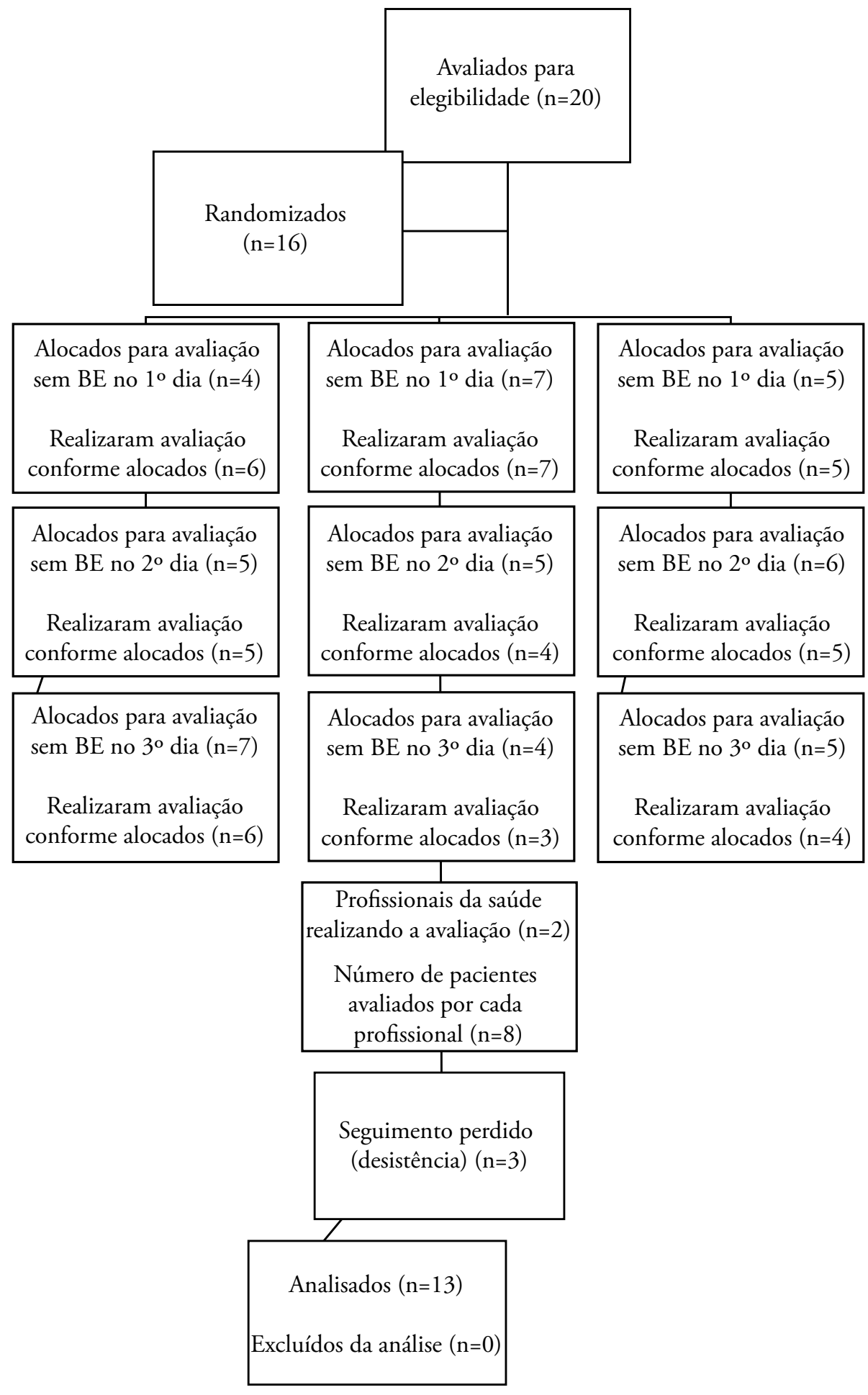

FIGURA 4 - Diagrama representando o fluxo dos participantes em cada etapa do estudo.

\section{Análise de dados}

\section{Eletromiografia}

Os dados eletromiográficos foram processados por meio de rotinas desenvolvidas em ambiente Matlab $\left(\right.$ Mathworks ${ }^{\odot}$ ). Para o cálculo do pico de ativação do sinal eletromiográfico durante o teste de força muscular, foi criado um envelope linear por meio da retificação de onda inteira e suavizaçáo dos sinais, com a utilização de filtro passa baixa de $4^{\mathrm{a}}$ ordem, com frequência de corte de $10 \mathrm{~Hz}^{5}$. Foi analisado o pico de ativação muscular dos músculos RF, VM e VL nas três condiçóes propostas. 


\section{Torque muscular}

Os dados de torque muscular foram processados em rotinas desenvolvidas em ambiente Matlab (Mathworks ${ }^{\circledR}$ ), utilizando um filtro Butterworth de $4^{\mathrm{a}}$ ordem com frequência de corte de $15 \mathrm{~Hz}^{21}$. Os dados de torque foram normalizados pela massa corporal dos voluntários. O pico de torque foi determinado pelo maior valor de torque obtido após o início da contração muscular.

\section{Análise estatística}

A análise estatística foi realizada por meio do software PASW statistics $18.0^{\circ}$ (SPSS). Após verificação da normalidade e homogeneidade dos dados foi utilizado o teste estatístico ANOVA Medidas Repetidas considerando fator condição (3 níveis), utilizando o post hoc de Bonferroni. Foi adotado o nível de significância de $\mathrm{p}<0,05$.

\section{Resultados}

O teste ANOVA Medidas Repetidas mostrou que não houve diferença significativa entre as condições sem BE, BE placebo e BE ativação ( $p=0,556$, $\mathrm{F}=0,936$ ), conforme TABELA 2.

TABELA 2 - Médias e desvios padrão da ativação eletromiográfica do quadríceps e torque isométrico dos extensores de joelho para as condições avaliadas

\begin{tabular}{|c|c|c|c|c|}
\hline & \multicolumn{3}{|c|}{ PICO DE ATIVAÇÃO MUSCULAR } & \multirow{2}{*}{$\begin{array}{l}\text { TORQUE } \\
\left(\mathrm{N} / \mathrm{Kg}^{-1}\right)\end{array}$} \\
\hline & $\begin{array}{c}\text { RETO } \\
\text { FEMORAL }\end{array}$ & $\begin{array}{c}\text { VASTO } \\
\text { MEDIAL }\end{array}$ & $\begin{array}{c}\text { VASTO } \\
\text { LATERAL }\end{array}$ & \\
\hline SEM BE & $100,00 \pm 0,00$ & $100,00 \pm 0,00$ & $100,00 \pm 0,00$ & $1,97 \pm 0,52$ \\
\hline BE ATIVAÇÃO & $112,03 \pm 33,89$ & $99,02 \pm 32,28$ & $121,18 \pm 25,57$ & $2,07 \pm 0,57$ \\
\hline BE PLACEBO & $109,78 \pm 28,58$ & $95,82 \pm 37,13$ & $109,05 \pm 24,31$ & $2,06 \pm 0,49$ \\
\hline
\end{tabular}

Valores de média \pm desvio padrão

\section{Discussão}

Este estudo investigou o efeito da BE na ativação eletromiográfica do quadríceps e torque extensor de joelho em indivíduos com OAJ, por meio da aplicação do método de facilitação neuromuscular. Os nossos resultados refutam nossa hipótese inicial de que a BE promoveria aumento da ativação muscular e do pico de torque isométrico dos extensores do joelho nessa populaçáo.

Segundo seu criador, o uso da BE promove o aumento da circulação sanguínea e linfática, o que melhora a função muscular, possivelmente aumentando sua ativação8. Entretanto os achados deste estudo não mostraram alteração no recrutamento muscular do quadríceps após a aplicação da BE. Nossos dados estáo de acordo com os estudos de Lins et al. (2013) e Yeung et al. (2014), que avaliaram o desempenho neuromuscular do quadríceps e não observaram diferenças significativas após a aplicação da bandagem sobre os músculos RF, VM e VL e no VM oblíquo respectivamente, em indivíduos jovens e saudáveis, sugerindo que a ausência de efeito da BE era em decorrência dos participantes não apresentarem comprometimento muscular ${ }^{10,22}$. Entretanto, nosso demonstra ausência de efeito da BE para alteração da ativação muscular ou força mesmo envolvendo uma populaçáo com déficit neuromuscular dos extensores do joelho.

Com relação à ativaçáo muscular, nossos dados não concordam com alguns estudos pregressos que mostraram um aumento na atividade eletromiográfica após aplicação da $\mathrm{BE}^{22,23}$. Possíveis explicaçôes para essa discordância podem estar relacionadas à diferença metodológica para aplicação da BE e variáveis analisadas. Hsu et al. (2009) aplicaram a BE no músculo trapézio inferior em atletas com Síndrome do Impacto, e seus resultados se devem ao fato de que a BE promoveu um suporte funcional à articulação 
escapulo-umeral, favorecendo indiretamente a ativação muscular ${ }^{23}$. SLUPIK et al. (2007) investigaram o efeito da $\mathrm{BE}$ sobre o músculo VM e mostraram que a atividade bioelétrica aumentou 24 horas após a aplicação da BE e permaneceu durante 48 horas após sua remoção, sugerindo que um aumento gradual do tônus pode começar várias horas após a aplicação da bandagem ${ }^{24}$. Além disso, seus achados não mostraram aumento no pico de torque após 10 minutos de aplicação, tal como em nosso estudo, no qual foi avaliado apenas o efeito agudo da BE, o que pode ter contribuído para não obtermos diferenças significativas nas avaliaçóes.

O estudo de Serra et al. (2015) avaliaram jogadores de futebol profissional saudáveis com relação à força dos extensores do joelho imediatamente, e 24 horas após a aplicação da $\mathrm{BE}$ em comparação com a aplicação de Micropore, não demonstrando diferença significativa em ambas as avaliaçóes ${ }^{25}$. Esse achado contribuiu para o entendimento de que independente da população, saudável ou com alteraçôes musculoesqueléticas, a $\mathrm{BE}$ não foi capaz de gerar aumento imediato do pico de torque.

Os resultados conflitantes encontrados na literatura a respeito do efeitos proporcionados pelo uso da $\mathrm{BE}$ pode estar relacionado com as diferentes formas e tensóes de aplicação da BE, já que diferentes técnicas podem fornecer diferentes intensidades de estímulos táteis.
Além disso, a metodologia empregada para análise do efeito da BE também pode mostrar influência nos resultados, sendo que variáveis temporais da ativação muscular se mostram mais sensíveis para essa técnica, incluindo o tempo para atingir o pico de torque, tempo de reaçáo, tempo pré-motor e tempo motor.

De acordo com a revisão sistemática de literatura de Morris et al. (2012), existe uma limitada a moderada evidência de que a $\mathrm{BE}$ não é mais efetiva, clinicamente, do que uma bandagem comum/ placebo, e não existem evidências suficientes para apoiar seu uso sobre outras modalidades na prática clínica $^{26}$. Apesar do uso da BE ser comum na prática clínica, faltam evidências na literatura que suportem os efeitos inerentes à sua aplicação e expliquem os mecanismos de atuação dessa técnica. Sugere-se que novos estudos sejam feitos para analisar o efeito crônico da BE na população com OAJ, mensurando a quantidade de tensão aplicada na bandagem através do comprimento da fita na régua.

Podemos concluir que a bandagem elástica não foi capaz de proporcionar aumento no recrutamento muscular do quadríceps e do torque isométrico dos extensores de joelho em indivíduos com OAJ. Novos estudos devem ser feitos na perspectiva de compreender o efeito da BE para essa população.

\section{Abstract \\ Effect of elastic taping on muscle activation and isometric torque of the knee extensors in subjects with Osteoarthritis}

Osteoarthritis is a chronic joint disease characterized by a progressive degeneration of articular cartilage, resulting in functional impairment. Among the affected joints, the knee is the most affected due to mechanical overload imposed on the joint in daily activities. The knee osteoarthritis is considered one of the most disabling forms of the disease. The aim of this study was to investigate the effect of elastic taping (ET) in quadriceps muscle activation and in isometric torque of knee extensors in subjects with knee osteoarthritis. The study included 13 volunteers ( $64.5 \pm 7.2$ years) female, with radiological diagnosis of knee osteoarthritis. Data collection procedures were carried out in three days, with an interval of seven days between them. The volunteers were evaluated in three different conditions: without ET, placebo-ET and activation-ET. Evaluations of knee extensor isometric torque and electromyographic activation of the rectus femoris, vastus medialis and vastus lateralis through surface electromyography were performed. Repeated Measures ANOVA test was used for statistical analysis and level of significance was set at $p<0.05$. The results showed no significant difference compared muscle activation and torque between the conditions analyzed $(p=0.556)$. It follows that ET has not been capable of promoting a change in muscle recruitment and quadriceps isometric torque in subjects with knee osteoarthritis.

KEYWORDS: Electromyography; Physical therapy; Muscle Strength; Joints. 


\section{Notas}

a. Estudo desenvolvido na Universidade Estadual Paulista "Júlio de Mesquita Filho" (UNESP) pelo Departamento de Fisioterapia e Terapia Ocupacional.

b. Parecer de aprovação no Comitê de Ética em Pesquisa da FFC/UNESP Marília - protocolo no 1.299.741/2015.

\section{Limitações do estudo}

Apesar da BE ter sido aplicada sempre pelo mesmo fisioterapeuta com uma técnica padronizada, a quantidade de tensão não foi quantificada.

O nível de dor foi controlado por meio da EVA $(<3 \mathrm{~cm})$, entretanto esse é um meio de avaliação subjetivo e isso pode ter influenciado no resultado uma vez que a dor e a inibição artrogênica são fatores que interferem no recrutamento muscular e na produção de força.

Este estudo avaliou apenas o efeito agudo da BE e em indivíduos do gênero feminino com OAJ pelo fato desta patologia ser mais frequente em mulheres, porém isto limita a extensão desses dados para outras populaçóes. Ademais, as variáveis temporais da ativação muscular não foram analisadas.

\section{Fonte de financiamento}

Financiamento próprio.

\section{Declaração de conflito de interesses}

Os autores declaram que não há conflito de interesses associado a esse estudo.

\section{Referências}

1. Anan M, Shinkoda K, Suzuki k, Yagi M, Kito N. Dynamic frequency analyses of lower extremity muscles during sit-to-stand motion for the patients with knee osteoarthritis. PLoS One. 2016;11(1):e0147496.

2. Sociedade Brasileira de Reumatologia. Osteoartrite (Artrose). São Paulo: Comissão de Osteoartrite da Sociedade Brasileira de Reumatologia; 2011.

3. Bagnato GL, Miceli G, Marino N, Sciortino D, Bagnato GF. Pulsed electromagnetic fields in knee osteoarthritis: a double blind, placebo-controlled, randomized clinical trial. Rheumatology (Oxford). 2016;55(4):755-62.

4. Rice DA, McNair PJ, Lewis GN, Dalbeth N. Quadriceps arthrogenic muscle inhibition: the effects of experimental knee joint effusion on motor cortex excitability. Arthritis Res Ther. 2014;16(6):502.

5. Marques NR, LaRoche DP, Hallal CZ, Crozara LF, Morcelli MH, Karuka AH, et al. Association between energy cost of walking, muscle activation, and biomechanical parameters in older female fallers and non-fallers. Clin Biomech. 2013;28(3):330-6.

6. Segal NA, Glass NA, Torner J, Yang M, Felson DT, Sharma L, et al. Quadriceps weakness predicts risk for knee joint space narrowing in women in the MOST cohort. Osteoarthritis Cartilage. 2010;18(6):769-75.

7. Bennell KL, Hunt MA, Wrigley TV, Hunter DJ, McManus FJ, Hodges PW et al. Hip strengthening reduces symptoms but not knee load in people with medial knee osteoarthritis and varus malalignment: a randomised controlled trial. Osteoarthritis Cartilage. 2010;18(5):621-

8. Kase K, Wallis J, Kase T. Clinical therapeutic applications of the kinesio taping method. 2a. ed. Albuquerque: Kinesio Taping Association; 2003. 
9. Kase K, Tatsuyuki H, Tomoko O. Development of kinesio tape. Kinesio taping perfect manual. Albuquerque: Kinesio Taping Association; 1996.

10. Lins CA, Neto FL, Amorim AB, Macedo LB, Brasileiro JS. Kinesio Taping does not alter neuromuscular performance of femoral quadriceps or lower limb function in healthy subjects: randomized, blind, controlled, clinical trial. Man Ther. 2013;18(1):41-5.

11. Anandkumar S; Sudarshan S, Nagpal P. Efficacy of Kinesio Taping on isokinetic quadriceps torque in knee osteoarthritis: a double blinded randomized controlled study. Physiother Theory Pract. 2014;30(6):375-83.

12. Kellgren JH, Lawrence JS. Radiological assessment of osteoarthrosis. Ann Rheum Dis. 1957;16(4):494-502.

13. Hallal CZ, Marques NR, Spinoso DH, Vieira ER, Gonçalves M. Electromyographic patterns of lower limb muscles during apprehensive gait in younger and older female adults. J Electromyogr Kinesiol. 2013;23(5):1145-9.

14. Chang HY, Wang CH, Chou KY, Cheng SC. Could forearm Kinesio Taping improve strength, force sense, and pain in baseball pitchers with medial epicondylitis? Clin J Sport Med. 2012;22(4):327-33.

15. Larsen AH, Puggaard L, Hämäläinen U, Aagaard P. Comparison of ground reaction forces and antagonist muscle coactivation during stair walking with ageing. J Electromyogr Kinesiol. 2008;18(4):568-80.

16. Costa RA, Oliveira LM, Watanabe SH, Jones A, Natour J. Isokinetic assessment of the hip muscles in patients with osteoarthritis of the knee. Clinics. 2010;65(12):1253-9.

17. Hartmann A, Knols R, Murer K, Bruin ED. Reproducibility of an isokinetic strength-testing protocol of the knee and ankle in older adults. Gerontology. 2009;55(3):259-68.

18. Hall SJ. Biomecânica Básica. 6a. ed. Rio de Janeiro: Guanabara Koogan; 2013.

19. Gonçalves M, Barbosa F. Análise de parâmetros de força e resistência dos músculos eretores da espinha lombar durante a realização de exercício isométrico em diferentes níveis de esforço. Rev Bras Med Esporte. 2005; 11(2):109-14.

20. Hermens JH, Freriks B, Disselhorst-Klug C, Rau G. Development of recommendations for SEMG sensors and sensor placement procedures. J Electromyogr Kinesiol. 2000;10(5): 361-74.

21. Crozara LF, Morcelli MH, Marques NR, Hallal CZ, Spinoso DH, Neto AFA, et al. Motor readiness and joint torque production in lower limbs of older women fallers and non-fallers. J Electromyogr Kinesiol. 2013;23(5):1131-8.

22. Yeung SS, Yeung EW, Sakunkaruna Y, Mingsoonngern S, Hung WY, Fan YL, Iao HC. Acute effects of Kinesio Taping on knee extensor peak torque and electromyographic activity after exhaustive isometric knee extension in healthy young adults. Clin J Sport Med. 2015;25(3):284-90.

23. Hsu YH, Chen WY, Lin HC, Wang WT, Shih YF. The effects of taping on scapular kinematics and muscle performance in baseball players with shoulder impingement syndrome. J Electromyogr Kinesiol. 2009;19(6):1092-9.

24. Slupik A, Dwornik M, Bialoszewski D, Zych E. Effect of Kinesio Taping on bioelectrical activity of vastus medialis muscle: preliminary report. Ortop Traumatol Rehabil. 2007;9(6):644-51.

25. Serra MV, Vieira ER, Brunt D, Goethel MF, Gonçalves M, Quemelo PR. Kinesio Taping effects on knee extension force among soccer players. Braz J Phys Ther. 2015;19(2):152-8.

26. Morris D, Jones D, Ryan H, Ryan CG. The clinical effects of Kinesio ${ }^{\circledR}$ Tex taping: a systematic review. Physiother Theory Pract. 2013;29(4):259-70.

\author{
ENDEREÇO \\ Deborah Hebling Spinoso \\ Universidade Estadual Paulista \\ Departamento de Fisioterapia e \\ Terapia Ocupacional \\ Av. Higino Muzzi Filho, 737 \\ 17525-900 - Marília - São Paulo - BRASIL \\ E-mail: deborahebling@yahoo.com.br
}

Recebido para publicação: 25/02/2016

$1^{\text {a }}$ Revisão: 18/05/2016

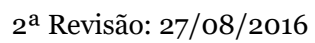

Aceito: 03/11/2016 